\title{
Accurate Calculations of U(1) Lattice Gauge Theory
}

\author{
S.J. Baker, R.F. Bishop ${ }^{* \dagger}$ and N.J. Davidson \\ Department of Physics, University of Manchester Institute of Science and Technology (UMIST), \\ P O Box 88, Manchester M60 1QD, United Kingdom
}

We apply the powerful coupled cluster method (CCM) to the $U(1)$ lattice gauge model in $2+1$ dimensions, using as a reference state for our analysis a self-consistent "mean-field" state which includes a large class of one-body correlations. We consider up to local two-body correlations and obtain results which are on a par with those from the best alternative techniques. Our results strongly indicate that the ground state of the $U(1)$ system in $2+1$ dimensions is rather weakly correlated.

\section{Introduction}

The coupled cluster method (CCM) is widely recognized as being one of the most powerful, most widely applied, and numerically most accurate of all available techniques of microscopic quantum many-body theory. The U(1) model in $2+1$ dimensions, although comparatively simple, is strongly nonperturbative, and hence provides a strong test for the CCM.

The Hamiltonian formulation that the CCM uses has several advantages over that of the more common Lagrangian formulations. In particular, the Hamiltonian approach can remove the need to extrapolate the results obtained on finite lattices to the infinite lattice limit. It also leaves the time dimension continuous, so that the extrapolation to the continuum limit need only be performed in the spatial dimensions.

In the initial work $[1,2]$, the strong-coupling ground state or chromoelectreic vacuum was used as the reference state, while in our recent paper [3] we have used a more complicated "meanfield" reference state in which a certain class of one-body correlations is already included. The great advantage of this new reference state is that it provides exact results for the single-plaquette Mathieu problem in both the strong- and weak-

\footnotetext{
"E-mail: R.F.Bishop@umist.ac.uk

†This work is supported by a research grant from EPSRC (Great Britain).

FPresent address: Department of Physics, University of Pretoria, Pretoria 0002, South Africa.
}

coupling limits without using the behaviour in these limits as prior knowledge.

This work is arranged as follows. We first describe the CCM in Sec. 2 as it is applied to a general system, and then in Sec. 3 we specialize the description to the present U(1) model. In Sec. 4 we present our results, and Sec. 5 contains our conclusions and discussion of further work.

\section{The Coupled Cluster Method}

The CCM constructs the exact ground state from some normalized reference state $|\Phi\rangle$ and a corresponding complete set of multiconfigurational creation and destruction operators $\left\{C_{I}^{\dagger}, C_{I}\right\}$ in the following way:

$\left|\Psi_{0}\right\rangle=e^{S}|\Phi\rangle,\left\langle\tilde{\Psi}_{0}\right|=\langle\Phi| \tilde{S} e^{-S}$,

where

$S=\sum_{I} s_{I} C_{I}^{\dagger}, \tilde{S}=1+\sum_{I} \tilde{s}_{I} C_{I}$.

The many-body operators $C_{I}$ and their Hermitian counterparts $C_{I}^{\dagger}$ are constructed as appropriate products of single-body operators. They are defined with respect to $|\Phi\rangle$ as a vacuum state, so that $C_{I}|\Phi\rangle=0=\langle\Phi| C_{I}^{\dagger}$.

Note that the bra state is not manifestly constrained to be the Hermitian conjugate of the ket state. The Hellmann-Feynman theorem, which states that

$$
\frac{\partial}{\partial \lambda}\langle\tilde{\Psi}(\lambda)|H(\lambda)| \Psi(\lambda)\rangle=\left\langle\tilde{\Psi}(\lambda)\left|\frac{\partial H(\lambda)}{\partial \lambda}\right| \Psi(\lambda)\right\rangle
$$


where $\langle\tilde{\Psi}(\lambda) \mid \Psi(\lambda)\rangle=1$, and $|\Psi(\lambda)\rangle$ and $\langle\tilde{\Psi}(\lambda)|$ are adjoint energy eigenstates, is thus conserved for all approximations of $S$ and $\tilde{S}$.

To perform practical calculations we need to approximate $S$ and $\tilde{S}$, and the most common method to do so is the so-called SUBn scheme. We can write $S$ as

$S=S_{1}+S_{2}+S_{3}+\ldots$,

where $S_{i}$ contains only $i$-body terms. The SUBn approximation is where we set $S_{i}=0$ for $i>n$, thus only keeping up to, and including, the $n-$ body terms. $\tilde{S}$ is similarly approximated by

$\tilde{S}=1+\tilde{S}_{1}+\tilde{S}_{2}+\tilde{S}_{3}+\ldots$.

The expectation value of the Hamiltonian of the system is given by

$\langle H\rangle=\left\langle\Phi\left|\tilde{S} e^{-S} H e^{S}\right| \Phi\right\rangle=E_{0}$,

where $E_{0}$ is the ground-state energy. Using variational techniques we can solve for the coefficients $\left\{s_{I}, \tilde{s}_{I}\right\}$ by requiring that $\langle H\rangle$ is stationary with respect to each coefficient. Thus we have

$$
\begin{aligned}
& \frac{\partial\langle H\rangle}{\partial \tilde{s}_{I}}=0=\left\langle\Phi\left|C_{I} e^{-S} H e^{S}\right| \Phi\right\rangle, \\
& \frac{\partial\langle H\rangle}{\partial s_{I}}=0=\left\langle\Phi\left|\tilde{S} e^{-S}\left[H, C_{I}^{\dagger}\right] e^{S}\right| \Phi\right\rangle .
\end{aligned}
$$

Equations (6) and (7) shows that in order to calculate the ground-state energy one needs only the coefficients $\left\{s_{I}\right\}$,

$E_{0}=\left\langle\Phi\left|e^{-S} H e^{S}\right| \Phi\right\rangle$.

An important feature of the CCM is that the expression $e^{-S} H e^{S}$ may be easily expanded using the nested commutator expansion,

$$
e^{-S} H e^{S}=H+[H, S]+\frac{1}{2 !}[[H, S] S]+\ldots
$$

For many Hamiltonians this expansion either terminates or can be resummed to a finite function.

Excited states $\left|\Psi_{\lambda}\right\rangle$ can be created by letting an excitation operator $X^{\lambda}$ act on the ground state,

$$
\begin{aligned}
& \left|\Psi_{\lambda}\right\rangle=X^{\lambda}\left|\Psi_{0}\right\rangle=X^{\lambda} e^{S}|\Phi\rangle, \\
& X^{\lambda}=\sum_{I} x_{I}^{\lambda} C_{I}^{\dagger} .
\end{aligned}
$$

The excitation energy gaps $\varepsilon^{\lambda}$ are given by the solutions to the eigenvalue equation,

$$
\left\langle\Phi\left|C_{I} e^{-S}\left[H, X^{\lambda}\right] e^{S}\right| \Phi\right\rangle=\varepsilon^{\lambda}\left\langle\Phi\left|C_{I} X^{\lambda}\right| \Phi\right\rangle .
$$

Only the ground-state coefficients $\left\{s_{i}\right\}$ are needed to obtain these.

\section{The CCM Applied to the U(1) Model.}

The U(1) lattice Hamiltonian, in (1+1 or) $2+1$ dimensions, can be written solely in terms of the elementary plaquette variables $B_{p}$ as

$$
\begin{aligned}
H=\sum_{p} & {\left[-2 \frac{\partial^{2}}{\partial B_{p}^{2}}+\lambda\left(1-\cos B_{p}\right)\right] } \\
+ & \frac{1}{2} \sum_{p, \rho} \frac{\partial^{2}}{\partial B_{p} \partial B_{p+\rho}},
\end{aligned}
$$

where the sum over $p$ is over the $N_{p}$ plaquettes and that over $\rho$ is over the nearest-neighbour plaquettes to $p$.

We construct our states using only gaugeinvariant terms, namely functions of the plaquette variables $B_{p}$. Such states are automatically gauge-invariant. For our reference state $|\Phi\rangle$ we use the 'mean-field' state [3] defined as

$|\Phi\rangle=e^{T}|0\rangle$,

where

$T=t \sum_{p} \cos B_{p}$,

and $t$ is a parameter to be determined by some external means (e.g., variationally). The state $|0\rangle$ represents the strong-coupling $(\lambda \rightarrow 0)$ chromoelectric vacuum.

The inner product of $\left\langle\tilde{g}\left(\left\{B_{p}\right\}\right)\right|$ and $\left|f\left(\left\{B_{p}\right\}\right)\right\rangle$ is defined as

$$
\langle\tilde{g} \mid f\rangle=\prod_{p} \int_{-\pi}^{\pi}\left(\frac{d B_{p}}{I_{0}(2 t) 2 \pi}\right) \tilde{g} f,
$$

where $I_{0}(2 t)$ is the zeroth-order modified Bessel function,

$$
I_{n}(2 t)=\int_{-\pi}^{\pi} \cos n x e^{2 t \cos x} d x
$$

Thus, $\left\langle\Phi\left|\cos n B_{p}\right| \Phi\right\rangle=R_{n}$ where $R_{n} \equiv$ $I_{n}(2 t) / I_{0}(2 t)$. The parameters $\left\{R_{n}\right\}$ are readily 
evaluated in terms of the simply derived recurrence relation,

$R_{n-1}-R_{n+1}=\frac{n}{t} R_{n}$,

using backwards recurrence with $R_{0}=1$.

We take as our operators, $\left\{C_{I}^{\dagger}\right\}$ and $\left\{C_{I}\right\}$, products of single-plaquette operators corresponding to the strong coupling limit $(\lambda \rightarrow 0)$, namely $\left\{\left(\cos m B_{p}-R_{m}\right) ; m=0,1,2, \ldots\right\}$ and $\left\{\sin m B_{p} ; m=1,2,3, \ldots\right\}$. The presence of the $R_{m}$ term in the even parity operators is required to make them orthogonal.

For $S$ expressed as in Eq. (4) we have the expansions,

$$
\begin{aligned}
S_{1}= & \sum_{n=1}^{\infty} \sum_{p} a_{p}(n)\left(\cos n B_{p}-R_{n}\right) \\
S_{2}= & \sum_{n_{1}, n_{2}=1}^{\infty} \sum_{p_{1}, p_{2}}^{\prime}\left[b_{p_{1}, p_{2}}\left(n_{1}, n_{2}\right)\left(\cos n_{1} B_{p_{1}}-R_{n_{1}}\right)\right. \\
\quad & \times\left(\cos n_{2} B_{p_{2}}-R_{n_{2}}\right) \\
& \left.+c_{p_{1}, p_{2}}\left(n_{1}, n_{2}\right) \sin n_{1} B_{p_{1}} \sin n_{2} B_{p_{2}}\right]
\end{aligned}
$$

with similar expressions for $\left\{S_{n}, n>2\right\}$ and for $\left\{\tilde{S}_{n}\right\}$. The prime on the plaquette summation in Eq. (21) indicates the omission of the term $p_{1}=p_{2}$. Only even parity operators are used as $H$ conserves parity.

\subsection{The 'Mean-Field' Approximation}

The simplest approximation we can make is that of the 'mean-field' scheme, which corresponds to the SUB0 level, with $S=0$ and $\tilde{S}=1$. This yields the expression,

$$
\langle H\rangle=\lambda+(t-\lambda) R_{1},
$$

which only depends on $t$. If we minimize $\langle H\rangle$ with respect to $t$, we thus have the consistency condition

$\lambda=t+\frac{R_{1}}{R_{1}^{\prime}}$.

If we neglect the last (interplaquette coupling) term in Eq. (14), the U(1) Hamiltonian reduces to one for $N_{p}$ independent plaquettes, each of which is described by the Mathieu Hamiltonian. The above 'mean-field' scheme gives the correct behaviour for the Mathieu problem in both the strong- and weak-coupling limits. In the rest of this work $t$ is fixed by the solution of Eq. (23).

\subsection{Ground-State Equations}

We now introduce the LSUB2 level of approximation, including only the one-body and nearest-neighbour two-body correlations. The ground-state equations corresponding to Eq. (7) are generated by.

$$
\begin{aligned}
0=\langle & \left\langle\Phi\left|\left(\cos m B_{q}-R_{m}\right) e^{-S} H e^{S}\right| \Phi\right\rangle, \\
0= & \langle\Phi|\left(\cos m_{1} B_{q_{1}}-R_{m_{1}}\right) \\
& \times\left(\cos m_{2} B_{q_{2}}-R_{m_{2}}\right) e^{-S} H e^{S}|\Phi\rangle, \\
0=\langle & \left\langle\Phi\left|\sin m_{1} B_{q_{1}} \sin m_{2} B_{q_{2}} e^{-S} H e^{S}\right| \Phi\right\rangle ; \\
& \quad m, m_{1}, m_{2}=1,2, \ldots ;\left|q_{1}-q_{2}\right|=1 .
\end{aligned}
$$

We further approximate to the LSUB2 $(n)$ level where

$$
\begin{gathered}
a_{p}(i)=0 ; i>n, \\
b_{p_{1}, p_{2}}(i, j)=0=c_{p_{1}, p_{2}}(i, j) ; i+j>n,
\end{gathered}
$$

and $p_{1}$ and $p_{2}$ are limited to nearest-neighbour plaquettes. These equations generate a set of $N(n)$ second-order coupled nonlinear equations for the number, $N(n)$, of independent $\operatorname{LSUB2}(n)$ coefficients. It is not difficult to show that $N(n)=\frac{1}{2} n^{2}+n$ if $n$ is even, and $N(n)=$ $\frac{1}{2}\left(n^{2}+2 n-1\right)$ if $n$ is odd. Furthermore, as $H$ contains second-order differential terms, it is clear that the expansion of Eq. (10) terminates in this case after the third term.

\subsection{Excited States}

There are two groups of excitations for the $\mathrm{U}(1)$ model, distinguished by their parity under the transformation $\left\{B_{p}\right\} \rightarrow\left\{-B_{p}\right\}$, and thus we require the two excitation correlation operators denoted by $X^{e}$ and $X^{o}$ for the even and odd parity operators respectively. They both have the form $X=X_{1}+X_{2}+\ldots$, where $X_{n}$ is the $n$-plaquette term of $X$. For convenience we use the same level of approximation for $X$ as we do for $S$.

The CCM gives us a set of general eigenvalue equations (13) to be solved for the excited states. Of note is the similarity between $X^{e}$ and $S$, which means that the left side of the even eigenstate equations can be reproduced by taking the 
Table 1

Ground-state energy density $\left(E_{0} / N_{p}\right)$ of the $\mathrm{U}(1)$ model in $2+1$ dimensions.

\begin{tabular}{llccccccc}
\hline$\lambda$ & 0.5 & 1 & 1.5 & 2.0 & 4.0 & 9.0 & 16.0 & 200 \\
\hline SUB1 & 0.43912 & 0.77243 & 1.03159 & 1.24302 & 1.85974 & 2.86879 & 3.87066 & 14.01600 \\
LSUB2(2) & 0.43868 & 0.76800 & 1.01853 & 1.21891 & 1.79290 & 2.73900 & 3.69427 & 13.40277 \\
LSUB2(3) & 0.43867 & 0.76769 & 1.01722 & 1.21604 & 1.78469 & 2.73355 & 3.69473 & 13.40505 \\
LSUB2(4) & 0.43867 & 0.76773 & 1.01759 & 1.21731 & 1.79217 & 2.74684 & 3.70538 & 13.40548 \\
LSUB2(5) & 0.43867 & 0.76774 & 1.01764 & 1.21754 & 1.79411 & 2.75007 & 3.70694 & 13.40547 \\
LSUB2(6) & 0.43867 & 0.76774 & 1.01765 & 1.21755 & 1.79431 & 2.75031 & 3.70682 & 13.40547 \\
LSUB2(7) & 0.43867 & 0.76774 & 1.01765 & 1.21755 & 1.79432 & 2.75033 & 3.70684 & 13.40547 \\
GFMC [4] & 0.43868 & $0.76745(1)$ & $1.01635(2)$ & $1.21505(1)$ & $1.793(1)$ & & & \\
$t$-expan.a [5] & $0.7675(4)$ & & & $1.796(2)$ & $2.763(3)$ & $3.724(12)$ & \\
$t$-expan.b [5] & $0.7689(9)$ & & & $1.796(10)$ & $2.757(12)$ & $3.712(20)$ & \\
\hline
\end{tabular}

ground-state equations and replacing each coefficient of $S$ with the corresponding coefficient of $X^{e}$, one at a time. This is useful as a check on our algebra and in the numerical evaluation of the equations.

\section{Results}

The results for the ground-state energy density $E_{0} / N_{p}$ are given in Table 1 for the U(1) model in $2+1$ dimensions. The results of a Green's function Monte Carlo calculation (GFMC) [4] and those of a calculation using the $t$-expansion method [5] are shown for comparison. As can be seen, our results show good agreement and converge rapidly for all values of $\lambda$.

The results that we obtain for the mass gap energies, which are the results for the first oddparity excitation energy, show the correct exponential decay, even at the SUB1 level where one would expect to find the Mathieu problem results in the absence of the self-consistency obtained from the use of the 'mean-field' state with the value of $t$ fixed by Eq. (23).

The results for the even excitation energy at the SUB1 level reproduce the results for the Mathieu problem, whereas the LSUB2 approximation gives better results for values of $\lambda$ below about 1 but fails to give significant improvement at larger values. Preliminary work at higher levels of approximation indicates that to get improvement we need to include higher-order plaquette correlations rather than longer-ranged two-plaquette correlations.

\section{Conclusions}

The CCM produces high accuracy results for the $U(1)$ model in $2+1$ dimensions for relatively little computational effort, the results shown being produced in about an hour on a p.c. The relatively low number of correlation coefficients needed to produce these results indicates that the ground state is rather weakly correlated in this 'mean-field' basis.

Some work on $\mathrm{U}(1)$ in $3+1$ dimensions has been done in Ref. [6]. The ability to form closed surfaces of plaquettes in this case creates additional difficulties, and higher-order calculations still need to be done.

We also intend to apply the CCM to nonabelian gauge theories such as $\mathrm{SU}(2)$.

\section{REFERENCES}

1. R.F. Bishop, A.S. Kendall, L.Y. Wong, and Y. Xian, Phys. Rev. D 48, 887 (1993).

2. R.F. Bishop. and Y. Xian, Acta Phys. Pol. B 3, 541 (1993).

3. S.J. Baker, R.F. Bishop and N.J. Davidson, Phys. Rev. D 53, 2610 (1996)

4. C.J. Hamer, K.C. Wang, and P.F. Price, Phys. Rev. D 50, 4693 (1994).

5. C.J. Morningstar, Phys. Rev. D 46, 824 (1992).

6. N.J. Davidson and R.F. Bishop, Nucl. Phys. B (Proc. Suppl.) 42, 817 (1995). 